\title{
On the Largest Zeroes of Orthogonal Polynomials for Certain Weights
}

\author{
By D. S. Lubinsky* and A. Sharif
}

Abstract. The asymptotic growth of the largest zero of the orthogonal polynomials for the weights $W(x)=|x|^{b} \exp \left(-k|\log | x||^{c}\right)$ is investigated.

1. Introduction. Freud [3], [4] investigated the largest zeroes of orthogonal polynomials for weights on $(-\infty, \infty)$. Nevai and Dehesa [5] studied the sums of powers of zeroes of orthogonal polynomials. Here we investigate the asymptotic growth of the largest zeroes for the weights

$$
\begin{array}{r}
W(x)=|x|^{b} \exp \left(-k|\log | x||^{c}\right), \quad x \in(-\infty, \infty) \\
\quad \text { where } c>1 ; k>0 ; b \in(-\infty, \infty)
\end{array}
$$

and

$$
W(x)= \begin{cases}x^{b} \exp \left(-k|\log x|^{c}\right), & x \in(0, \infty), \\ 0, & x \in(-\infty, 0],\end{cases}
$$

where $c>1 ; k>0 ; b \in(-\infty, \infty)$.

When $c=2$ and $b=0$ in (1.2), $W(x)$ yields the Stieltjes-Wigert polynomials (Chihara [1, 2]), and Chihara [2] has remarked that very little is known about their zeroes.

2. Notation. Given a nonnegative measurable function $W(x)$ on $(-\infty, \infty)$ for which all moments

$$
\mu_{n}(W)=\int_{-\infty}^{\infty} x^{n} W(x) d x, \quad n=0,1,2, \ldots,
$$

exist, its orthogonal polynomials are

$$
p_{n}(W ; x)=\gamma_{n}(W) \prod_{j=1}^{n}\left(x-x_{j n}(W)\right), \quad n=0,1,2, \ldots
$$

satisfying

$$
\int_{-\infty}^{\infty} p_{n}(W ; x) p_{m}(W ; x) W(x) d x= \begin{cases}1, & m=n \\ 0, & m \neq n\end{cases}
$$

Received May 5, 1982.

1980 Mathematics Subject Classification. Primary 42C05, 33A65.

*Research supported by Lady Davis Fellowship. 
We let $X_{n}(W)=\max \left\{\left|x_{j n}(W)\right|: j=1,2, \ldots, n\right\}, n=1,2, \ldots$ Further, for each positive $t, \zeta_{t}$ denotes the smallest possible number (if it exists) such that

$$
\left(\zeta_{t}\right)^{t} W\left(\zeta_{t}\right)=\max \left\{x^{t} W(x): x \in(0, \infty)\right\}
$$

and assuming that

$$
\int_{0}^{\pi}|\log W(\zeta \cos \theta)| d \theta<\infty, \quad \zeta \in(0, \infty),
$$

we define

$$
G_{\zeta}(W)=\exp \left\{\pi^{-1} \int_{0}^{\pi} \log W(\zeta \cos \theta) d \theta\right\}, \quad \zeta \in(0, \infty)
$$

\section{The Largest Zero.}

LeMma 3.1. Let $W(x)$ be given by (1.1). Then

$$
\lim _{n \rightarrow \infty} \mu_{2 n}(W) /\left[d(2 n+b+1)^{(2-c) /(2(c-1))} \exp \left\{f(2 n+b+1)^{c /(c-1)}\right\}\right]=1,
$$

where $d=2\left\{2 \pi(c-1)^{-1}(k c)^{1 /(1-c)}\right\}^{1 / 2}$ and $f=(c-1)\left(c^{c} k\right)^{1 /(1-c)}$.

Proof.

$$
\begin{aligned}
\mu_{2 n}(W) & =2 \int_{1}^{\infty} x^{2 n+h} \exp \left(-k(\log x)^{c}\right) d x+O\left(n^{1}\right) \\
& =2\left(c k^{1 / c}\right)^{-1} \int_{0}^{\infty} \exp \left(-v+v^{1 / c} X\right) v^{1 / c-1} d v+O\left(n^{-1}\right),
\end{aligned}
$$

where $X=(2 n+b+1) k^{-1 / c}$ and $x=\exp \left((v / k)^{1 / c}\right)$. Now apply the asymptotic formula for the integral in (3.1), given in Olver [6, p. 84, Ex. 7.3].

Following is our main result.

THEOREM 3.2. Let $W(x)$ be given by (1.1). Then

$$
\begin{gathered}
\lim _{n \rightarrow \infty}\left(\frac{k c}{2 n}\right)^{1 /(c-1)} \log X_{n}(W)=1 \\
\lim _{n \rightarrow \infty}\left(\frac{k c}{2 n}\right)^{1 /(c-1)} \log \left\{\gamma_{n-1}(W) / \gamma_{n}(W)\right\}=1
\end{gathered}
$$

Proof. (i) By Lemma 3 in Freud [3, p. 95],

$$
\begin{aligned}
\log X_{n}(W) & \geqslant\left(\log \mu_{2 n-2}(W)-\log \mu_{2 n-4}(W)\right) / 2 \\
& =(f / 2)\left\{(2 n+b-1)^{c /(c-1)}-(2 n+b-3)^{c /(c-1)}\right\}+O\left(n^{-1}\right) \\
& =(f / 2)(2 n)^{c /(c-1)}\left\{c(c-1)^{-1} n^{-1}+O\left(n^{-2}\right)\right\}+O\left(n^{-1}\right) \\
& =(2 n / k c)^{1 /(c-1)}+O\left(n^{(2-c) /(c-1)}\right) .
\end{aligned}
$$

Next, for any $\zeta>0$ and $A>1$, Theorem 2 in Freud [4, p. 52] shows that

$$
X_{n}(W) \leqslant A \zeta+\frac{4}{3 \pi}\left(\frac{2}{\zeta}\right)^{2 n-1} G_{\zeta}^{-1}(W) \int_{A \zeta}^{\infty} x^{2 n-1} W(x) d x .
$$


Freud states this under the additional assumption that $W(x)$ is positive in $(-\infty, \infty)$, but his proof is valid if (2.2) holds. It is easily seen that for some positive constant $K_{0}$, independent of $\zeta$,

$$
G_{\zeta}(W) \geqslant K_{0}^{-1} W(\zeta), \quad \zeta \in(0, \infty)
$$

Then taking $\zeta=\zeta_{2(n+s)}$ and $A=2^{n / s}$ where $s \in(0, \infty)$, we obtain, from (2.1), (3.3) and (3.4),

$$
\begin{aligned}
X_{n}(W) & \leqslant A \zeta_{2(n+s)}+\frac{4 K_{0}}{3 \pi}\left(\frac{2}{\zeta_{2(n+s)}}\right)^{2 n-1} \zeta_{2(n+s)}^{2(n+s)} \int_{A \zeta_{2(n+s)}}^{\infty} x^{-1-2 s} d x \\
& =\zeta_{2(n+s)}\left[2^{n / s}+K_{0}(3 \pi s)^{-1}\right] .
\end{aligned}
$$

Next, for large $t \in(0, \infty), \zeta_{t}$ is a root of $d\left[x^{t} W(x)\right] / d x=0$ so $\log \zeta_{t}=$ $[(t+b) / k c]^{1 /(c-1)}$. Taking $s=n^{\delta}$ in (3.5) where

$$
0<\delta<1 \text { and } 1-\delta(c-1)^{-1}
$$

we obtain

$$
\log X_{n}(W) \leqslant\left[\left(2 n+2 n^{\delta}+b\right) / k c\right]^{1 /(c-1)}+n^{1-\delta} \log 2+o(1) .
$$

The result follows from (3.2), (3.6) and (3.7).

(ii) follows from (i) and Theorem 1 in Freud [3, p. 91].

Since

$$
\left\{X_{n}(W)\right\}^{m} \leqslant \sum_{j=1}^{n}\left|x_{j n}(W)\right|^{m} \leqslant n\left\{X_{n}(W)\right\}^{m}, \quad m>0, n=1,2, \ldots,
$$

we deduce that, for $m>0$,

$$
\lim _{n \rightarrow \infty}\left(\frac{k c}{2 n}\right)^{1 /(c-1)} \log \left\{\sum_{j=1}^{n}\left|x_{j n}(W)\right|^{m}\right\}=m,
$$

which provides a contrast to the results of Nevai and Dehesa [5, Theorem 1].

Corollary 3.3. Let $W(x)$ be given by (1.2). Then the conclusions (i), (ii) of Theorem 3.2 remain true.

Proof. Let

$$
W^{*}(x)=|x| W\left(x^{2}\right)=|x|^{2 b+1} \exp \left(-k_{1}|\log | x||^{c}\right), \quad x \in(-\infty, \infty),
$$

where $k_{1}=k 2^{c}$. Then, by Theorem 3.2,

$$
\begin{gathered}
\lim _{n \rightarrow \infty}\left(\frac{k_{1} c}{4 n}\right)^{1 /(c-1)} \log X_{2 n}\left(W^{*}\right)=1, \\
\lim _{n \rightarrow \infty}\left(\frac{k_{1} c}{4 n}\right)^{1 /(c-1)} \log \left\{\gamma_{2 n-j-1}\left(W^{*}\right) / \gamma_{2 n-j}\left(W^{*}\right)\right\}=1, \quad j=0,1 .
\end{gathered}
$$

Further, the substitution $x=u^{2}$ yields $p_{n}\left(W ; u^{2}\right)=p_{2 n}\left(W^{*} ; u\right)$ and hence

$$
X_{n}(W)=\left\{X_{2 n}\left(W^{*}\right)\right\}^{2} ; \quad \gamma_{n}(W)=\gamma_{2 n}\left(W^{*}\right),
$$

and the conclusions follow from (3.8) and (3.9). 
For real $b$, and fixed positive $k$, let

$$
W_{b}(x)= \begin{cases}k \pi^{-1 / 2} x^{h} \exp \left(-k^{2}(\log x)^{2}\right), & x \in(0, \infty), \\ 0, & x \in(-\infty, 0] .\end{cases}
$$

Wigert [7] explicitly found $p_{n}\left(W_{0} ; x\right), n=1,2, \ldots$, while Chihara [2] constructed discrete solutions of the moment problem corresponding to $W_{0}$, which provided some information regarding the distribution of $\left\{x_{n j}\left(W_{0}\right)\right\}_{n, j}$. Using the relation

$$
W_{b}(x)=\alpha^{b^{2}} W_{0}\left(x / \alpha^{2 b}\right), \quad x \in(-\infty, \infty),
$$

where $\alpha=\exp \left(1 / 4 k^{2}\right)$, it follows that

$$
p_{n}\left(W_{b} ; x\right)=\alpha^{-b(b+2) / 2} p_{n}\left(W_{0} ; x / \alpha^{2 h}\right), \quad n=1,2, \ldots,
$$

and hence the results of Wigert [7] and Chihara [2] for $W_{0}(x)$ generalize to $W_{b}(x)$, any $b \in(-\infty, \infty)$.

Department of Mathematics

Technion-Israel Institute of Technology

32000 Haifa, Israel

1. T. S. Chimara, An Introduction to Orthogonal Polynomials, Gordon and Breach, New York, 1978.

2. T. S. Chihara, "A characterization and a class of distribution functions for the Stieltjes-Wigert polynomials," Canad. Math. Bull., v. 13, 1970, pp. 529-532.

3. G. FreUd, "On the greatest zero of an orthogonal polynomial. I," Acta Sci. Math. (Hungar.), v. 34. 1973, pp. 91-97.

4. G. Freud, “On the greatest zero of an orthogonal polynomial. II," Acta Sci. Math. (Hungar. ), v. 36. 1974, pp, 49-54.

5. P. G. Nevai \& J. S. Dehesa, "On asymptotic average properties of zeroes of orthogonal polynomials," SIAM J. Math. Anal., v. 10, 1979, pp. 1184-1192.

6. F. W. J. Olver, Asymptotics and Special Functions, Academic Press, New York, 1974.

7. S. WigerT, "Sur les polynomes orthogonaux et l'approximation des fonctions continues," Ark. Mat. Astronom. Fysik, v. 17, 1923. 